\title{
Efecto de NPK en la Producción de Citrus aurantifolia Swingle V. Sutil en Sinchal, Santa Elena.
}

\author{
Néstor Orrala B. ${ }^{(1)}$, Araceli Solís L. ${ }^{(2)}$, María Tomalá C. ${ }^{(3)}$ \\ Centro de Investigaciones Agropecuarias ${ }^{(1,2)}$ \\ Facultad de Ciencias Agrarias ${ }^{(3)}$ \\ Universidad Estatal Península (UPSE) de Santa Elena \\ Campus La Libertad, vía principal Santa Elena- La Libertad \\ La Libertad - Ecuador \\ norralab@hotmail.com,norrala@upse.edu.ec
}

\begin{abstract}
Resumen
La presente investigación evaluó variables agronómicas y de calidad postcosecha en el limón sutil bajo el efecto de diferentes dosis de nitrógeno fosforo y potasio, solas o en combinación aplicados al suelo durante un año calendario, dividida en tres dosis en la comuna Sinchal, cantón Santa Elena. Se utilizó un diseño de completamente aleatorio. Las variables evaluadas fueron de producción: diámetro del fruto, peso del fruto, grosor de la cáscara, rendimiento por árbol y de calidad pos-cosecha: sólidos solubles totales (Grados Brix), porcentaje de acidez títulable, índice de madurez, porcentaje de jugo y análisis económico. Las variables agronómicas y de calidad postcosecha giran alrededor de lo obtenido por investigadores de América Latina y del Ecuador. Tukey 5 \% señala diferencias significativas en el rendimiento por planta durante el año analizado. El tratamiento testigo alcanzó $39,43 \mathrm{~kg} /$ planta a diferencia del tratamiento 12, que obtuvo 98,11 kg/planta. El análisis económico estableció que el mejor tratamiento fue el $8\left(N_{750} P_{150} K_{300}\right)$ con una tasa de retorno marginal 267,06\%.
\end{abstract}

Palabras claves: Limón sutil, fertilizantes, rendimiento, calidad postcosecha.

\begin{abstract}
The investigation evaluated agronomic variables and post-harvest quality in key lime under the effect of different doses of nitrogen, phosphorus and potassium, alone or in combination applied to the soil during a calendar year, divided into three doses in Sinchal, Santa Elena province. It was used a randomized design. The production variables evaluated were: fruit diameter, fruit weight, shell thickness, yield per plant and post-harvest quality: total soluble solids (Brix), labeled acid percentage, maturity index, juice content and economic analysis. The agronomic variables and post harvest quality were around the results obtained by researchers from Latin America and Equator. Tukey $5 \%$ indicate significant differences in the yield per plant during the year under review. The control treatment reached $39,43 \mathrm{~kg} /$ plant in contrast to the treatment 12 , which was $98,11 \mathrm{~kg} /$ plant. The economic analysis determined that the best treatment was $8\left(P_{150} K_{300} N_{750}\right)$ with a return rate marginal 267, $06 \%$.
\end{abstract}

Keywords: Mexican lime, fertilization, yield, postharvest quality 


\section{INTRODUCCIÓN}

Los cítricos ocupan el primer lugar de la producción de frutos a nivel mundial. De acuerdo a investigaciones de la FAO la producción mundial de limas y limones en el año 2001 fue 10,9 millones de toneladas. Los principales productores son México (14 \%), India (13\%), Argentina (11\%), España (9 \%), Estados Unidos (8\%), Irán (8\%) e Italia (5\%). Se exportaron 1,6 millones de toneladas de limas y limones en el año 2000, por un valor de US\$ 726 millones. Los principales exportadores fueron España (31\%), México (17\%), Argentina (13\%), Turquía (10 \%), Estados Unidos (7 \%), e Italia (2 \%). En el año 2001, Ecuador logró exportar más de 9000 t (1).

En Ecuador se cultivan limones "sutil" y "Tahití para el consumo local y exportación, respectivamente. Entre ambas especies existían $4 \quad 405$ ha en monocultivo, en 3257 unidades de producción agropecuarias (UPAs), principalmente en las provincias de Pichincha, Manabí y Guayas; en las dos últimas se concentran la producción de limón Tahití, en un $60 \%$ asociada con otros cultivos (2). De las 350 hectáreas de cultivos de limón sutil en la frontera orense, al igual que las 1200 ha sembradas en Portoviejo y Santa Ana, en Manabí, la zona de mayor producción del cítrico, no más del $6 \%$ de las plantaciones están tecnificadas (3).

Según el Instituto de Investigaciones Agropecuarias, Estación Portoviejo, esta puede ser la razón de que los intentos por exportar la variedad 'criolla' del cítrico conocida como sutil ó lima mexicana- no ha tenido éxito. Hasta abril del 2008 se habían exportado sólo 11000 kilos de limón (no detalla si la fruta salió procesada o fresca). En el 2005 se exportó más, unos 102760 kilos, casi en su totalidad a Estados Unidos y durante el 2004, apenas 21000 kilos (4).

El factor riego eleva el costo de producción de limón, y también el hecho de que, como con cualquier otro cítrico de temporada, el precio del limón de igual manera varía según la oferta. En enero -en lo más alto de la cosecha- el valor de la malla (saco de 1200 unidades) cae a cuatro dólares. Sin embargo cuando escasea en la frontera, se paga hasta 12 dólares la malla más pequeña, de mil unidades.

En la provincia de Santa Elena, el fenómeno de El Niño de 1982, destruyó todas las plantaciones citrícolas existentes en la parte norte. Al ser, la actividad citrícola un medio de subsistencia, los campesinos volvieron a sembrar, principalmente limón criollo. Actualmente, en la zona de influencia del río Valdivia, (parroquias Manglaralto y Colonche, respectivamente) hay 130 ha de limón criollo, distribuidas en unidades de producción agropecuarias desde 0,6 a 10 ha, injertadas por lo general sobre limón rugoso (5).

La mayoría de los citricultores no reciben asistencia técnica de ninguna institución pública o privada y el manejo de las plantaciones es de carácter artesanal. Los rendimientos son deficientes y están muy por debajo de su potencial de producción. Siguiendo paquetes tecnológicos adecuados para la zona del valle del río Valdivia, se puede dejar atrás estas condiciones. Aumentar la producción es posible debido a la mayor actividad fisiológica de los cítricos durante el año, lo cual permite tener al mismo tiempo flores y frutos en diferentes etapas de desarrollo, a diferencia de las zonas subtropicales donde hay un periodo de descanso y la producción es estacionaria.

Por lo general, los cítricos necesitan como promedio 100 g de nitrógeno, 50 g de fósforo y 100 g de potasio por planta y por año (6). Estas cantidades de elementos nutritivos son referenciales; en Ecuador, concretamente en la provincia de Santa Elena, poco o nada es la información técnica que se encuentra, por lo que es importante investigar la influencia de varias dosis de fertilizantes en la producción anual de Citrus aurantifolia v. sutil.

La investigación se planteó como objetivo general: determinar la influencia de NPK en la producción de Citrus aurantifolia Swingle v. sutil en SinchalBarcelona, cantón Santa Elena; como específicos: a) Establecer la dosis óptima de fertilizantes en la producción de limón sutil, b) Determinar las principales características agronómicas y las propiedades físicas químicas de los frutos en cada uno de los tratamientos, c) Realizar un análisis económico de los tratamientos, según metodología del CIMMYT.

\section{Materiales y métodos}

El experimento se llevó a efecto en la Granja Zoilita, ubicada en la comuna Sinchal, cantón Santa Elena, altura $47 \mathrm{msnm}$. topografía plana; capa arable de origen aluvial, con temperaturas bien diferenciadas de mayo a diciembre con promedio de $24{ }^{\circ} \mathrm{C}$ y entre los meses de enero a abril, $27,2{ }^{\circ} \mathrm{C}$; humedad relativa entre 74 y $82 \%$ y precipitación alrededor de 100-250 mm (diciembre - mayo): coordenadas geográficas Latitud Sur 1 ${ }^{\circ} 56$ '9” y Longitud Oeste 8041'20”.

Suelo pH 7,9 ligeramente alcalino; nitrógeno bajo, 12 ppm; fosforo alto, $42 \mathrm{ppm}$; potasio $14 \mathrm{meq} / 100 \mathrm{ml}$ considerado alto; calcio $14 \mathrm{meq} / 100 \mathrm{ml}$ alto; magnesio, alto con 3,1 meq/100 ml; los microelementos, azufre $102 \mathrm{ppm}$; zinc y cobre 0,7 ppm bajo; el hierro 4 ppm considerado bajo; manganeso bajo con 5,0 y boro 2,30 ppm, alto.

En el experimento se utilizó plantas de Citrus aurantifolia $v$. sutil, de aproximadamente 14 años, injertadas sobre limón rugoso (Citrus jambhiri,), patrón tolerante a tristeza, exocortis y xyloporosis; muy sensible a Phytophthora sp y sensible a nemátodos. Muy vigoroso, induce a elevada productividad, sistema radical muy fuerte con muchas raíces laterales. Distancia de siembra 5,5 x 5,5 m.

Se utilizó un diseño completamente aleatorio (DCA), donde los tratamientos fueron diferentes niveles de $\mathrm{N}$, 
NP y NPK más un testigo; hubo cuatro réplicas. Cada unidad experimental estuvo conformada por una planta, Cuadro 1.

Cuadro 1. Tratamientos, gramos/planta/año

\begin{tabular}{|c|c|}
\hline Tratamientos & Niveles de NPK \\
\hline $\mathrm{T}_{1}$ & $\mathrm{~N}_{0} \mathrm{P}_{0} \mathrm{~K}_{0}$ \\
\hline $\mathrm{T}_{2}$ & $\mathrm{~N}_{750} \mathrm{P}_{0} \mathrm{~K}_{0}$ \\
\hline $\mathrm{T}_{3}$ & $\mathrm{~N}_{1000} \mathrm{P}_{0} \mathrm{~K}_{0}$ \\
\hline $\mathrm{T}_{4}$ & $\mathrm{~N}_{1250} \mathrm{P}_{0} \mathrm{~K}_{0}$ \\
\hline $\mathrm{T}_{5}$ & $\mathrm{~N}_{750} \mathrm{P}_{150} \mathrm{~K}_{0}$ \\
\hline $\mathrm{T}_{6}$ & $\mathrm{~N}_{1000} \mathrm{P}_{150} \mathrm{~K}_{0}$ \\
\hline $\mathrm{T}_{7}$ & $\mathrm{~N}_{1250} \mathrm{P}_{150} \mathrm{~K}_{0}$ \\
\hline $\mathrm{T}_{8}$ & $\mathrm{~N}_{750} \mathrm{P}_{150} \mathrm{~K}_{300}$ \\
\hline $\mathrm{T}_{9}$ & $\mathrm{~N}_{1000} \mathrm{P}_{150} \mathrm{~K}_{300}$ \\
\hline $\mathrm{T}_{10}$ & $\mathrm{~N}_{1250} \mathrm{P}_{150} \mathrm{~K}_{300}$ \\
\hline $\mathrm{T}_{11}$ & $\mathrm{~N}_{1000} \mathrm{P}_{150} \mathrm{~K}_{200}$ \\
\hline $\mathrm{T}_{12}$ & $\mathrm{~N}_{1000} \mathrm{P}_{150} \mathrm{~K}_{400}$ \\
\hline $\mathrm{T}_{13}$ & $\mathrm{~N}_{1000} \mathrm{P}_{150} \mathrm{~K}_{500}$ \\
\hline
\end{tabular}

Los fertilizantes aplicados durante el año del experimento están detallados en el cuadro 2.

Cuadro 2. Dosis fertilizantes comerciales, gramos /planta/año

\begin{tabular}{|c|c|c|c|c|c|}
\hline \multirow{2}{*}{ Tratamientos } & \multicolumn{3}{|c|}{ Primer ciclo } & \multicolumn{2}{c|}{$\begin{array}{c}\text { Segundo y tercer } \\
\text { ciclo }\end{array}$} \\
\cline { 2 - 6 } & Urea & Urfos & Hydrocomplex & Urea & $\begin{array}{c}\text { Sulfato } \\
\text { de } \\
\text { potasio }\end{array}$ \\
\hline $\mathrm{T}_{1}-\mathrm{N}_{0} \mathrm{P}_{0} \mathrm{~K}_{0}$ & 0 & 0 & 0 & 0 & 0 \\
\hline $\mathrm{T}_{2}-\mathrm{N}_{250} \mathrm{P}_{0} \mathrm{~K}_{0}$ & 543,5 & 0 & 0 & 543,50 & 0 \\
\hline $\mathrm{T}_{3}-\mathrm{N}_{333} \mathrm{P}_{0} \mathrm{~K}_{0}$ & 723,9 & 0 & 0 & 723,90 & 0 \\
\hline $\mathrm{T}_{4}-\mathrm{N}_{417} \mathrm{P}_{0} \mathrm{~K}_{0}$ & 906,5 & 0 & 0 & 906,50 & 0 \\
\hline $\mathrm{T}_{5}-\mathrm{N}_{250} \mathrm{P}_{150} \mathrm{~K}_{0}$ & 413,3 & 333,3 & 0 & 543,50 & 0 \\
\hline $\mathrm{T}_{6}-\mathrm{N}_{333} \mathrm{P}_{150} \mathrm{Ko}_{0}$ & 593,6 & 333,3 & 0 & 723,90 & 0 \\
\hline $\mathrm{T}_{7}-\mathrm{N}_{417} \mathrm{P}_{150} \mathrm{Ko}_{0}$ & 776,3 & 333,3 & 0 & 906,52 & 0 \\
\hline $\mathrm{T}_{8}-\mathrm{N}_{250} \mathrm{P}_{150} \mathrm{~K}_{100}$ & 321,28 & 197,5 & 555,5 & 543,50 & 222,22 \\
\hline $\mathrm{T}_{9}-\mathrm{N}_{333} \mathrm{P}_{150} \mathrm{~K}_{100}$ & 501,71 & 197,5 & 555,5 & 723,90 & 222,22 \\
\hline $\mathrm{T}_{10}-\mathrm{N}_{417} \mathrm{P}_{150} \mathrm{~K}_{100}$ & 684,32 & 197,5 & 555,5 & 906,50 & 222,22 \\
\hline $\mathrm{T}_{11}-\mathrm{N}_{333} \mathrm{P}_{150} \mathrm{~K}_{67}$ & 532,06 & 242,4 & 372,2 & 723,90 & 148,89 \\
\hline $\mathrm{T}_{12}-\mathrm{N}_{333} \mathrm{P}_{150} \mathrm{~K}_{133}$ & 471.43 & 152,73 & 738,8 & 723,90 & 295,56 \\
\hline $\mathrm{T}_{13}-\mathrm{N}_{333} \mathrm{P}_{150} \mathrm{~K}_{167}$ & 440,21 & 106,6 & 927,7 & 723,90 & 371,11 \\
\hline
\end{tabular}

\subsection{Variables experimentales}

De producción: Diámetro del fruto, peso del fruto, grosor de la cáscara, rendimiento por árbol, rendimiento por hectárea.

De calidad pos-cosecha: Sólidos solubles totales (Grados Brix), porcentaje de acidez títulable, índice de madurez, porcentaje de jugo.

\section{Resultados y discusión}

\subsection{Variables agronómicas}

El cuadro 3, indica la significancia estadística de las variables agronómicas. Cuando se analizó el diámetro del fruto, Tukey $5 \%$ indica medias poblacionales iguales para todos los tratamientos. En cambio, en el peso del fruto existen dos grupos estadísticos, sobresaliendo los tratamientos 5 y 6 con 33,03 y 33,15 g; en el grosor de la cáscara se identifican tres grupos, ocupando el primer lugar el testigo absoluto. En estas variables, así mismo Tukey $5 \%$ encuentra significancia en los dos periodos analizados. Por último, la prueba de Tukey señala diferencias significativas en el rendimiento por planta durante el año. El tratamiento testigo alcanzó 39,43 kg/planta/año a diferencia del tratamiento 12, que obtuvo 98,11 $\mathrm{kg} /$ planta/año. Los coeficientes de variación de todas estas variables denotan la confiabilidad del experimento.

Cuadro 3. Significancia estadística de las variables agronómicas

\begin{tabular}{|c|c|c|c|c|}
\hline \multirow[b]{2}{*}{ Tratamientos } & \multicolumn{3}{|c|}{ Dos períodos } & \multirow[b]{2}{*}{$\begin{array}{l}\text { Rendimiento } \\
\mathrm{kg} / \text { planta/año }\end{array}$} \\
\hline & $\begin{array}{c}\text { Diámetro } \\
\text { del fruto } \\
\text { (cm) }\end{array}$ & $\begin{array}{l}\text { Peso del } \\
\text { fruto } \\
\text { (g) }\end{array}$ & $\begin{array}{l}\text { Grosor } \\
\text { de la } \\
\text { cascara } \\
(\mathrm{mm})\end{array}$ & \\
\hline $\mathrm{T}_{1} \quad \mathrm{~N}_{0} \mathrm{P}_{0} \mathrm{~K}_{0}$ & $3,76 \mathrm{a}$ & 28,93 a & $2,47 \mathrm{c}$ & 39,43 a \\
\hline $\mathrm{T}_{2} \quad \mathrm{~N}_{750} \mathrm{P}_{0} \mathrm{~K}_{0}$ & $3,81 \mathrm{a}$ & $31,67 \mathrm{ab}$ & $1,70 \mathrm{~b}$ & $79,48 \mathrm{bc}$ \\
\hline $\mathrm{T}_{3} \quad \mathrm{~N}_{1000} \mathrm{P}_{0} \mathrm{~K}_{0}$ & $3,78 \mathrm{a}$ & $31,53 \mathrm{ab}$ & $1,59 \mathrm{ab}$ & $56,91 \mathrm{ab}$ \\
\hline $\mathrm{T}_{4} \quad \mathrm{~N}_{1250} \mathrm{P}_{0} \mathrm{~K}_{0}$ & $3,91 \mathrm{a}$ & $32,40 \mathrm{ab}$ & $1,56 \mathrm{ab}$ & $90,23 \mathrm{c}$ \\
\hline $\mathrm{T}_{5} \quad \mathrm{~N}_{750} \mathrm{P}_{150} \mathrm{~K}_{0}$ & $3,8 \mathrm{a}$ & $33,03 \mathrm{~b}$ & $1,53 \mathrm{ab}$ & $71,77 \mathrm{bc}$ \\
\hline $\mathrm{T}_{6} \quad \mathrm{~N}_{1000} \mathrm{P}_{150} \mathrm{~K}_{0}$ & 3,89 a & $33,15 \mathrm{~b}$ & $1,51 \mathrm{ab}$ & 83,95 bc \\
\hline $\mathrm{T}_{7} \quad \mathrm{~N}_{1250} \mathrm{P}_{150} \mathrm{~K}_{0}$ & 3,85 a & $31,55 \mathrm{ab}$ & $1,44 \mathrm{ab}$ & 88,29 c \\
\hline $\mathrm{T}_{8} \quad \mathrm{~N}_{750} \mathrm{P}_{150} \mathrm{~K}_{300}$ & $3,89 \mathrm{a}$ & $32,42 \mathrm{ab}$ & $1,44 \mathrm{ab}$ & $93,84 \mathrm{c}$ \\
\hline $\mathrm{T}_{9} \quad \mathrm{~N}_{1000} \mathrm{P}_{150} \mathrm{~K}_{300}$ & $3,79 \mathrm{a}$ & $31,64 \mathrm{ab}$ & $1,49 \mathrm{ab}$ & $75,73 \mathrm{bc}$ \\
\hline $\mathrm{T}_{10} \quad \mathrm{~N}_{1250} \mathrm{P}_{150} \mathrm{~K}_{300}$ & $3,81 \mathrm{a}$ & $32,49 \mathrm{ab}$ & $1,43 \mathrm{ab}$ & $76,08 \mathrm{bc}$ \\
\hline $\mathrm{T}_{11} \quad \mathrm{~N}_{1000} \mathrm{P}_{150} \mathrm{~K}_{200}$ & $3,80 \mathrm{a}$ & $31,74 \mathrm{ab}$ & $1,31 \mathrm{a}$ & 72,65 bc \\
\hline $\mathrm{T}_{12} \quad \mathrm{~N}_{1000} \mathrm{P}_{150} \mathrm{~K}_{400}$ & $3,83 \mathrm{a}$ & $30,36 \mathrm{ab}$ & $1,47 \mathrm{ab}$ & $98,11 \mathrm{c}$ \\
\hline $\mathrm{T}_{13} \quad \mathrm{~N}_{1000} \mathrm{P}_{150} \mathrm{~K}_{500}$ & $3,74 \mathrm{a}$ & $30,52 \mathrm{ab}$ & $1,36 \mathrm{a}$ & 86 bc \\
\hline C.V. \% & 2,68 & 7,31 & 10,98 & 15,93 \\
\hline Media general & 3,82 & 31,65 & 1,56 & 77,88 \\
\hline $\begin{array}{l}\text { Periodo I } \\
\text { Periodo II }\end{array}$ & $\begin{array}{l}3,59 \mathrm{a} \\
4,04 \mathrm{~b}\end{array}$ & $\begin{array}{l}28,29 \mathrm{a} \\
35,01 \mathrm{~b}\end{array}$ & $\begin{array}{l}1,51 \mathrm{a} \\
1,61 \mathrm{~b}\end{array}$ & \\
\hline
\end{tabular}

El diámetro del fruto osciló entre 3,74 y 3,91cm, cifras que se sitúan alrededor de lo alcanzado por Caiza Briones JR. (7); Pérez Zamora O. (8); Cevallos Giler DA. y Cevallos Giler DA. y Cevallos Giler MO. (9) e INIAP (10) en diferentes investigaciones. En el peso del fruto, el testigo absoluto $\mathrm{N}_{0} \mathrm{P}_{0} \mathrm{~K}_{0}$ ocupa el último lugar; los demás tratamientos giran alrededor de lo alcanzado por otras investigaciones realizadas en el valle de Portoviejo.

La cáscara es más gruesa en el tratamiento 1 . Si bien es cierto, el contenido del fósforo es alto, es posible que su asimilación esté relacionada directamente con 
la falta de nitrógeno, pues su sinergismo es sumamente importante en la nutrición vegetal. Sin embargo, no es alarmante y no se sitúa, en lo que manifiesta Román H., CA. (11) al decir que la falta de fósforo produce frutos con cáscara gruesa y con un hueco en el centro. En cuanto al rendimiento Franciosi R. et al (12) dice: la existencia de cantidades de nitrógeno asimilable en el suelo antes y durante la floración, cuajado del fruto y comienzo del desarrollo del mismo, favorece notablemente la producción.

La significancia estadística de las variables de calidad postcosecha está señalada en el cuadro 4 . El análisis de la varianza no encontró diferencias significativas entre los tratamientos en porcentaje de jugo, grados Brix, índice de madurez pero si en acidez títulable, donde Tukey $5 \%$ denota dos grupos estadísticos. El coeficiente de variación, también muestran lo confiable del ensayo.

Cuadro 4. Significancia estadística de las variables de producción

\begin{tabular}{|c|c|c|c|c|}
\hline \multirow[b]{2}{*}{ Tratamientos } & \multicolumn{4}{|c|}{ Dos períodos } \\
\hline & $\begin{array}{l}\text { Porcentaje de } \\
\text { jugo }\end{array}$ & $\begin{array}{l}\text { Grados } \\
\text { Brix }\end{array}$ & $\begin{array}{c}\text { Acidez } \\
\text { títulable }\end{array}$ & $\begin{array}{l}\text { Índice de } \\
\text { madurez }\end{array}$ \\
\hline $\mathrm{T}_{1} \quad \mathrm{~N}_{0} \mathrm{P}_{0} \mathrm{~K}_{0}$ & $41,69 \mathrm{a}$ & $8,41 \mathrm{a}$ & $6,02 \mathrm{a}$ & $1,44 \mathrm{a}$ \\
\hline $\mathrm{T}_{2} \quad \mathrm{~N}_{750} \mathrm{P}_{0} \mathrm{~K}_{0}$ & 45,67 a & $8,4 \mathrm{a}$ & $6,72 \mathrm{ab}$ & $1,28 \mathrm{a}$ \\
\hline $\mathrm{T}_{3} \quad \mathrm{~N}_{1000} \mathrm{P}_{0} \mathrm{~K}_{0}$ & $44,5 \mathrm{a}$ & $8,74 \mathrm{a}$ & $7,40 \mathrm{~b}$ & $1,21 \mathrm{a}$ \\
\hline $\mathrm{T}_{4} \quad \mathrm{~N}_{1250} \mathrm{P}_{0} \mathrm{~K}_{0}$ & $48,84 \mathrm{a}$ & $8,33 \mathrm{a}$ & $6,61 \mathrm{ab}$ & $1,28 \mathrm{a}$ \\
\hline $\mathrm{T}_{5} \quad \mathrm{~N}_{750} \mathrm{P}_{150} \mathrm{~K}_{0}$ & 46,63 a & $8,18 \mathrm{a}$ & $6,41 \mathrm{ab}$ & $1,31 \mathrm{a}$ \\
\hline $\mathrm{T}_{6} \quad \mathrm{~N}_{1000} \mathrm{P}_{150} \mathrm{~K}_{0}$ & 42,25 a & $8,45 \mathrm{a}$ & $6,95 \mathrm{ab}$ & $1,26 \mathrm{a}$ \\
\hline $\mathrm{T}_{7} \quad \mathrm{~N}_{1250} \mathrm{P}_{150} \mathrm{~K}_{0}$ & 43,31 a & 8,09 a & $6,61 \mathrm{ab}$ & $1,26 \mathrm{a}$ \\
\hline $\mathrm{T}_{8} \quad \mathrm{~N}_{750} \mathrm{P}_{150} \mathrm{~K}_{300}$ & 42,58 a & $8,25 \mathrm{a}$ & $7,07 \mathrm{ab}$ & $1,2 \mathrm{a}$ \\
\hline $\begin{array}{ll}\mathrm{T}_{9} & \mathrm{~N}_{1000} \mathrm{P}_{150} \mathrm{~K}_{300}\end{array}$ & 43,49 a & $8,01 \mathrm{a}$ & $6,47 \mathrm{ab}$ & $1,29 \mathrm{a}$ \\
\hline $\begin{array}{ll}\mathrm{T}_{10} & \mathrm{~N}_{1250} \mathrm{P}_{150} \mathrm{~K}_{300} \\
\end{array}$ & 44,68 a & 8,13 a & $6,57 \mathrm{ab}$ & $1,27 \mathrm{a}$ \\
\hline $\mathrm{T}_{11} \quad \mathrm{~N}_{1000} \mathrm{P}_{150} \mathrm{~K}_{200}$ & 46,67 a & $8,46 \mathrm{a}$ & $6,78 \mathrm{ab}$ & $1,30 \mathrm{a}$ \\
\hline $\mathrm{T}_{12} \quad \mathrm{~N}_{1000} \mathrm{P}_{150} \mathrm{~K}_{400}$ & 43,83 a & $8,13 \mathrm{a}$ & $6,00 \mathrm{a}$ & $1,44 \mathrm{a}$ \\
\hline $\mathrm{T}_{13} \quad \mathrm{~N}_{1000} \mathrm{P}_{150} \mathrm{~K}_{500}$ & $45,03 \mathrm{a}$ & $8,11 \mathrm{a}$ & $6,56 \mathrm{ab}$ & $1,29 \mathrm{a}$ \\
\hline C.V. \% & 14,06 & 7,27 & 10,46 & 13,39 \\
\hline Media general & 44,55 & 8,28 & 6,63 & 1,29 \\
\hline $\begin{array}{l}\text { Periodo I } \\
\text { Periodo II }\end{array}$ & $\begin{array}{l}42,49 \text { a } \\
42,61 \mathrm{~b}\end{array}$ & $\begin{array}{l}8,12 \mathrm{a} \\
8,44 \mathrm{~b}\end{array}$ & $\begin{array}{l}5,72 \mathrm{a} \\
7,54 \mathrm{~b}\end{array}$ & $\begin{array}{l}1,52 \mathrm{~b} \\
1,08 \mathrm{a}\end{array}$ \\
\hline
\end{tabular}

Todas las variables de calidad postcosecha giran alrededor de lo obtenido por investigadores de América Latina; incluso el porcentaje de jugo del tratamiento testigo supera a los tratamientos que recibieron nitrógeno, fósforo y potasio. Posiblemente se explica en el contenido de elementos en el suelo, por ejemplo, fósforo y potasio. En esta variable los demás tratamientos no siguen un orden de acuerdo a las dosis recibidas, concordando con diversas investigaciones.

\subsection{Análisis económico}

El análisis marginal (Cuadro 5) destaca los tratamientos 5, 2, 6, 7, 4 y 8 que superan la tasa de retorno mínima aceptable, considerada $100 \%$. Esta metodología al no considerar el rendimiento, permitiría recomendar a los tratamientos que superan la tasa de retorno mínima aceptable. Para los pequeños productores inclusive el tratamiento 2, es muy válido. También el análisis marginal permite indicar que no siempre el tratamiento que alcanza el mejor resultado es el más recomendable. Igual tasa de retorno marginal se observa cuando se analiza la producción con las densidades de siembra $6 \begin{array}{lllllll}6 & \text { x } & 6 & \text { y } & 7 & \text { x } & 7 \text {, pero }\end{array}$ numéricamente los beneficios disminuyen a medida que aumenta la distancia.

Cuadro 5. Análisis marginal del experimento: "Efecto de NPK en la producción de Citrus Aurantifolia Swingle v. sutil en la zona Sinchal - Barcelona, Santa Elena, 2009. Dólares.

\begin{tabular}{|c|c|c|c|c|c|c|}
\hline \multicolumn{2}{|c|}{ Tratamientos } & $\begin{array}{c}\text { Costos } \\
\text { que } \\
\text { varian } \\
\text { (ha) }\end{array}$ & $\begin{array}{c}\text { Costos } \\
\text { marginales } \\
\text { (ha) }\end{array}$ & $\begin{array}{c}\text { Beneficios } \\
\text { netos } \\
\text { (ha) }\end{array}$ & $\begin{array}{c}\text { Beneficios } \\
\text { netos } \\
\text { marginales }\end{array}$ & $\begin{array}{c}\text { Tasa de } \\
\text { retorno } \\
\text { marginal }\end{array}$ \\
\hline$T_{1}$ & $\mathrm{~N}_{0} \mathrm{P}_{0} \mathrm{~K}_{0}$ & 0,0 & & 3187,86 & & \\
\hline & & & 277,26 & & 2336,90 & 842,855 \\
\hline$T_{5}$ & $\mathrm{~N}_{750} \mathrm{P}_{150} \mathrm{~K}_{0}$ & 277,26 & & 5524,76 & & \\
\hline & & & 24,06 & & 599,99 & 2493,606 \\
\hline$T_{2}$ & $\mathrm{~N}_{750} \mathrm{P}_{0} \mathrm{~K}_{0}$ & 301,32 & & 6124,75 & & \\
\hline & & & 75,93 & & 285,33 & 375,763 \\
\hline$T_{6}$ & $\mathrm{~N}_{1000} \mathrm{P}_{150} \mathrm{~K}_{0}$ & 377,25 & & 6410,08 & & \\
\hline & & & 101,25 & & 249,94 & 246,847 \\
\hline$T_{7}$ & $\mathrm{~N}_{1250} \mathrm{P}_{150} \mathrm{~K}_{0}$ & 478,50 & & 6660,02 & & \\
\hline & & & 24,06 & & 132,09 & 548,960 \\
\hline$T_{4}$ & $\mathrm{~N}_{1250} \mathrm{P}_{0} \mathrm{~K}_{0}$ & 502,56 & & 6792,10 & & \\
\hline & & & 79,55 & & 212,45 & 267,068 \\
\hline$T_{8}$ & $\mathrm{~N}_{750} \mathrm{P}_{150} \mathrm{~K}_{300}$ & 582,11 & & 7004,56 & & \\
\hline & & & 192,68 & & 152,63 & 79,215 \\
\hline$T_{12}$ & $\mathrm{~N}_{1000} \mathrm{P}_{150} \mathrm{~K}_{400}$ & 774,80 & & 7157,19 & & \\
\hline
\end{tabular}

\section{Conclusiones y recomendaciones}

\section{Conclusiones}

* Las variables agronómicas y de calidad postcosecha giran alrededor de lo alcanzado por algunas investigaciones de América Latina, lo que demuestra la influencia de los elementos estudiados en la producción.

* Los tratamientos 5, 2, 6, 7, 4, 8 superan la tasa de retorno mínima aceptable. Sin embargo el tratamiento 2 podría servir para pequeños productores.

* El único macroelemento limitante en la producción, en las condiciones agroecológicas del presente experimento, es el nitrógeno. En cuanto al fósforo, solo se requiere fertilización de mantenimiento.

\section{Recomendaciones}

* Realizar investigaciones que relacionen el contenido foliar de los macro y microelementos 
con el estado nutrimental del suelo y sirva como base de sistemas de fertilización.

* Implementar programas de investigación que consideren el efecto de los microelementos en la producción y en los parámetros de calidad del limón criollo.

* Implementar una base de datos que tributen a establecer NORMAS DRIS.

* Diseñar, considerando los resultados preliminares de esta investigación, programas de fertilización o paquetes tecnológicos para los citricultores, que permitan obtener mayor producción.

\section{Bibliografía}

1. FAO.FAOSTAT.2008.Limones

2. INEC. Censo agropecuario. 2000

3. Atcitrus. 2006. Disponible en http://www.atcitrus.com/noticia.asp?seccion=sec_ derecha\&id $=420$

4. Instituto Nacional Autónomo de Investigaciones Agropecuarias INIAP EC. 2007. "Desarrollo de prácticas de manejo de huertos frutícolas” Programa de fruticultura. 50503. Informe técnico anual. Portoviejo, EC.

5. Gonzabay, GX., y J.A. Galdea. 2004. Estudio Agro-socioeconómico de los citricultores del Valle del Río Valdivia, Parroquia Manglaralto, Cantón Santa Elena, Provincia del Guayas. Tesis Teclog. La Libertad, EC. Universidad Estatal Península de Santa Elena. 35 p.

6. Avilán L., y C. Rengifo. 1988. Los Cítricos. 1 ed. Caracas, América. C. p. 280-286

7. Caiza Briones, J.R. 1992. Estudio de fuentes y dosis de nitrógeno para el cultivo de limón criollo (Citrus aurantifolia L.) (Christm) Swingle, en la Provincia de Manabí. Tesis Ing. Agro. Portoviejo, EC. Universidad Técnica de Manabí. p. 40

8. Pérez Zamora, O. 2002. Evaluación de mejoradores del suelo en limón mexicano. Universidad Autónoma de Chapingo. Chapingo, Méx. Terra Latinoamericana. Vol. 20, No. 003. p 337-346

9. Cevallos Giler, DA. , y M.O. Cevallos Giler. 2007. Evaluación de tres dosis de ácido giberélico sobre la calidad de limón criollo (Citrus aurantifolia L.) Swing. En pre y post-cosecha. En el sitio Bígua del cantón Jama. Tesis Ing. Agr. Manabí, EC. Universidad Técnica de Manabí.

10. Instituto Nacional Autónomo de Investigaciones Agropecuarias INIAP. 1992. Climas, Suelos, Nutrición y Fertilización de Cultivos en el Litoral Ecuatoriano. Pichilingue, EC. Manual Técnico No. 26 p. 15

11. Román, H. CA. 1994. Instituto Colombiano Agropecuario ICA. Memorias del curso de actualización de Frutas Tropicales, CRECED.

12. Franciosi R. et al (1977). El cultivo de cítricos. Programa de Investigación en frutales.
Universidad Nacional Agraria - La Molina. Boletín técnico. PIF -No. 1

\section{Bibliografía no referenciada}

1. Federación Nacional de Cafeteros de Colombia. 1991. El cultivo de los cítricos. 8 ed. Colombia, Litografía Cafetera Ltda. 28 p

2. Instituto del Potasio y del Fósforo en Canadá, INPOFOS. s.f. Potasa.

3. International Plant Nutrición Institute IPNI (s.f.). Identificación de los Problemas Nutricionales en Cítricos. Síntomas de Deficiencia de Nutrientes. Disponible en www.ipni.net/ppiweb/gltamn.nsf/\$webindex/articl $\mathrm{e}=$ CDCF49FC05256ED6004D35F43EA2.- 21k

4. La Ville, E. 1979. Hilisation d' un Nouveau fongicide systemique: l'Aliette dans la lutte contre la gomose a Phytophtora des agrumes. p.41

5. Malavolta, E. 1981. Nutricao mineral e adubacao dos citrus. Ed. T. Yamada. Piracicaba. Instituto da Potasa. Boletín Técnico 5.. pp. 13 - 17.

6. Ministerio de Fomento. 1971. Anuario Estadístico. Caracas. s.e. Cuadro IX - 5. p 307

7. Olivera Arteaga, C. 1991. El cultivo de los cítricos en el valle de Huaral-Chancay. Fundación para el desarrollo del agropecuario "FUNDAGRO" Huaral, s.e. Perú

8. Pérez Zamora, O. y J. Orozco Romero 2001. Campo Experimental Tecomán. Instituto Nacional de Investigaciones Forestales, Agrícolas y Pecuarias. Tecomán, Colima, México. Terra Latinoamericana 22: 99-108.

9. Rodríguez, O. 1980. Nutricao e edubacao los citros. Em Micronutrientes. Campinas. s.n.t. Fundacao Cargill. p.107-124. 